\title{
Associations of Pre-Transplant Prescription Narcotic Use with Clinical Complications after Kidney Transplantation
}

\author{
Krista L. Lentine ${ }^{a, b} \quad$ Ngan N. Lam $^{c}$ Huiling Xiao ${ }^{a, b}$ Janet E. Tuttle-Newhall ${ }^{b}$ \\ David Axelrod $^{d}$ Daniel C. Brennan ${ }^{e}$ Vikas R. Dharnidharka $^{e}$ Hui Yuan $^{f}$ \\ Mustafa Nazzal $^{\text {b }}$ Jie Zhenga, e Mark A. Schnitzler ${ }^{b}$ \\ ${ }^{a}$ Center for Outcomes Research and ${ }^{b}$ Division of Abdominal Transplantation, Department of Surgery, Saint Louis \\ University School of Medicine, St. Louis, Mo., USA; ' Division of Nephrology, Western University, London, Ont., Canada; \\ dDivision of Abdominal Transplantation, Department of Surgery, Dartmouth Hitchcock Medical Center, Hanover, N.H., \\ USA; ${ }^{\mathrm{e}}$ Transplant Nephrology, Washington University School of Medicine, St. Louis, Mo., and ${ }^{\mathrm{f}}$ Department of Anesthesia, \\ Saint Louis University School of Medicine, St. Louis, Mo., USA
}

\section{Key Words}

Complications · Kidney transplantation · Opioid analgesics · Outcomes assessment · Pharmacy claims · Registries

\footnotetext{
Abstract

Background: The impact of narcotic use before kidney transplantation on post-transplant clinical outcomes is not well described. Methods: We examined integrated national transplant registry, pharmacy records, and Medicare billing claims to follow 16,322 kidney transplant recipients, of whom $28.3 \%$ filled a narcotic prescription in the year before transplantation. Opioid analgesic fills were normalized to morphine equivalents (ME) and expressed as $\mathrm{mg} / \mathrm{kg}$ exposures (approximate quartiles: 0.1-1.7, 1.8-5.4, 5.5-23.7, and $\geq 23.8 \mathrm{mg} / \mathrm{kg}$, respectively). Post-transplant cardiovascular, respiratory, neurological, accidents, substance abuse, and noncompliance events were identified using diagnosis codes on Medicare billing claims. Adjusted associations of ME level with post-transplant complications were quantified
}

by multivariate Cox regression. Results: The incidence of complications at 3 years post-transplant among those with the highest pre-transplant ME exposure compared to no use included: ventricular arrhythmias, 1.1 vs. $0.2 \%(p<0.001)$; cardiac arrest, 4.7 vs. $2.7 \%(\mathrm{p}<0.05)$; hypotension, 14 vs. $8 \%$ $(p<0.0001)$; hypercapnia, 1.6 vs. $0.9 \%(p<0.05)$; mental status changes, 5.3 vs. $2.7 \%(p<0.001)$; drug abuse/dependence, 7.0 vs. $1.7 \%$ ( $p<0.0001)$; alcohol abuse, 1.8 vs. $0.6 \%$ ( $p=0.0001)$; accidents, 0.9 vs. $0.3 \%(p<0.05)$; and noncompliance, 3.5 vs. $2.3 \%(p<0.05)$. In multivariate analyses, transplant recipients with the highest level of pre-transplant narcotic use had approximately 2 to 4 times the risks of posttransplant ventricular arrhythmias, mental status changes, drug abuse, alcohol abuse, and accidents compared with non-users, and $35-45 \%$ higher risks of cardiac arrest and hypotension. Conclusion: Although associations may reflect underlying conditions or behaviors, high-level prescription narcotic use before kidney transplantation predicts increased risk of clinical complications after transplantation.

(c) 2015 S. Karger AG, Basel

\section{KARGER 125}

(c) 2015 S. Karger AG, Basel

$0250-8095 / 15 / 0412-0165 \$ 39.50 / 0$ 


\section{Introduction}

In the last decade, prescription rates for opioid analgesics have increased dramatically in the United States [1, 2]. While these trends may reflect the increased awareness of acute and chronic pain as treatable conditions, many studies have shown correlations between the rise in prescription narcotic use and increasing rates of opioidrelated deaths, addictions, overdoses, adverse drug events such as somnolence and respiratory depression, as well as health care costs [1,3-5]. Patients with kidney failure may experience pain that is often multifactorial and related to their underlying comorbidities, such as polycystic kidney disease or diabetic neuropathy, complications of renal failure, such as calciphylaxis or renal osteodystrophy, and renal replacement therapy, such as vascular access use, steal syndrome during hemodialysis, or abdominal discomfort during peritoneal dialysis [6-8]. Prescription narcotic agents can be effective for the management of moderate-to-severe pain in patients with end-stage renal disease (ESRD) when applied as part of the World Health Organization's three-step analgesic ladder $[6,9]$. However, because kidney failure and dialysis can affect opioid pharmacokinetics and pharmacodynamics, patients with advanced renal disease may face a higher risk of adverse events due to the accumulation of the drugs and their metabolites, drug interactions with concurrent medications, and patient characteristics, such as age-related effects on drug metabolism in an older population [10, 11].

One cross-sectional study reported that the prevalence of pain in hemodialysis patients was similar to that in kidney transplant recipients (63 vs. $62 \%$ ), suggesting that kidney transplantation does not lead to a significant reduction in pain [12]. There is limited information on prescription narcotic use in patients with ESRD who subsequently receive a kidney transplant. Barrantes et al. performed a retrospective single-center study of 1,064 kidney transplant recipients followed for a median of 48 months (interquartile range [IQR], 34-63), and found that 452 recipients (42\%) had a history of chronic pain prior to transplantation and $108(10 \%)$ reported chronic opioid use prior to transplantation [13]. In the adjusted analyses, recipients with a history of chronic opioid use had a twofold higher risk of death, but similar risk of graft loss compared to matched recipients without a history of chronic opioid use. Recently, we examined a unique linkage of the national U.S. transplant registry with pharmacy claims data to follow 31,197 kidney transplant recipients, of whom 9,047 (29\%) filled a narcotic prescription in the year prior to transplant [14]. We found that the highest quartile of narcotic use prior to transplantation was associated with increased post-transplant death and graft loss (adjusted hazard ratio [aHR] 2.3; 95\% confidence interval [CI] 1.7-3.1; and aHR 1.7; 95\% CI 1.4-2.3, respectively) [14]. However, the clinical events that may mediate associations of narcotic use with post-transplant mortality and graft loss have not yet been examined in this population.

In the current study, we expanded our prior database of linked transplant registry and pharmacy fill records by merging administrative billing claims from Medicare that capture provider-reported clinical diagnoses. Using this integrated database, we sought to determine whether pretransplant narcotic exposure predicts post-transplant clinical complications including cardiovascular, respiratory, neurological, substance abuse, accidents, and noncompliance events.

\section{Methods}

Data Sources

The Organ Procurement and Transplantation Network (OPTN) data system captures information on all solid organ transplant recipients in the United States as submitted by OPTN member centers, including transplant date, demographic information, and annual follow-up surveys that query information on patient vital status and allograft function. The Health Resources and Services Administration (HRSA) and United States Department of Health and Human Services provide oversight to the activities of the OPTN contractor.

Pharmacy fill data were assembled by linking OPTN records for kidney transplant recipients with billing claims from a large pharmaceutical claims data (PCD) clearinghouse that captures prescription drug fill records including those reimbursed by public payers, private payers, and self-paid fills in the United States. The PCD comprises National Council for Prescription Drug Program (NCPDP) 5.1-format prescription claims aggregated from multiple sources including data clearinghouses, retail pharmacies, and prescription benefit managers for approximately $60 \%$ of retail pharmacy transactions in the United States. Individual claim records include the date of a given pharmacy fill with the National Drug Code (NDC) identifying agent and dosage. After Institutional Review Board and HRSA approvals, PCD records were linked with anonymous OPTN identification numbers for kidney transplant recipients using anonymous linkage tokens. We applied a de-identification strategy in which patient identifiers (last name, first name, sex, date of birth, and zip code of residence) were transformed before delivery to the Saint Louis University researchers with encryption technology from Management Science Associates, Inc. The Patient De-Identification Software employs multiple encryption algorithms in succession to guarantee that the resulting 'token' containing encrypted patient identifiers can never be decrypted. However, the algorithm yields the same results for a given set of data elements, such that linkages by unique anonymous tokens are possible. 
Medicare billing claims data include diagnostic and procedure codes for patients with Medicare fee-for-service primary or secondary insurance (service information is submitted to and tracked by Medicare for all Medicare beneficiaries, even if Medicare is not the primary payer). After regulatory approvals, beneficiary identifier numbers from Medicare's electronic databases were linked using Social Security Number, sex, and birthdates to unique OPTN identification numbers. Finally, patients with PCD data before transplant and Medicare claims data after transplant were selected for inclusion using anonymous OPTN identification numbers.

Because of the large sample size, the anonymity of the patients studied, and the nonintrusive nature of the research, a waiver of informed consent was granted per the Department of Health and Human Services Code of Federal Regulations (Title 45, Part 46, Paragraph 46.116). Analyses were performed using Health Information Portability and Accountability Act (HIPAA)-compliant, limited datasets from which all direct identifiers were removed. The Institutional Review Board of Saint Louis University approved this study.

\section{Sampling and Exposure Definitions}

We selected kidney transplant recipients with at least one year of captured pharmaceutical fill records before transplant and Medicare benefits beginning at the time of transplant. As PCD data were available beginning in 2005, patients with linked pre-transplant PCD data were transplanted beginning in 2006. The final analytic sample included patients transplanted in 2006 to 2008, based on overlap with the available Medicare claims data that ended in December 2008. Transplant recipient clinical and demographic characteristics, as well as characteristics of the donated organ and other transplant factors, were defined by the OPTN Transplant Candidate Registration (TCR) and Transplant Recipient Registration (TRR) forms (table 1).

Pharmacy fills for narcotic medications in the year prior to transplant were normalized to morphine equivalents (ME), according to conversion ratios as previously described [14]. Pretransplant $\mathrm{ME}$ were aggregated for each transplant recipient and expressed as $\mathrm{mg} / \mathrm{kg}$ ME exposure over the year. To maintain consistency with our prior study, we retained the same cut-points of annual ME among those who filled narcotic prescriptions pretransplant as: $0.1-1.7,1.8-5.4,5.5-23.7$, and $\geq 23.8 \mathrm{mg} / \mathrm{kg}$ [14]. Diagnoses of post-transplant ventricular arrhythmias, cardiac arrest, hypotension, hypercapnia, aspiration pneumonia, mental status change, drug abuse/dependence, alcohol abuse and associated complications, accidents, and noncompliance outcomes were defined by the identification of billing claims with corresponding International Classification of Diseases, 9th Revision, Clinical Modification (ICD-9-CM) diagnosis codes (online suppl. appendix 1; for all online suppl. material, see www.karger.com/doi/ $10.1159 / 000377685)$.

\section{Statistical Analyses}

Data management and analyses were performed with Statistical Analysis Software (SAS) for Windows software, version 9.3 (SAS Institute Inc., Cary, N.C., USA). Distributions of clinical and demographics traits among patients with each level of pre-transplant narcotic exposure, compared with no narcotic use, were compared by the $\chi^{2}$ test.

The incidence of each clinical complication, and a composite of any study complication, was estimated at 3 years post-transplant according to level of pre-transplant ME exposure by the KaplanMeier method. The log-rank test was used to assess the statistical significance of differences in unadjusted incidence across narcotic use levels. At-risk time for all models was censored for the following reasons: loss to follow-up, end of Medicare enrollment, end of study (December 31,2008), death for patients without concurrent study complication on date of death, or 3 years post-transplant.

Propensity scores for the likelihood of pre-transplant narcotic use were estimated by logistic regression as previously described [14]. Adjusted associations of ME with each post-transplant complication (adjusted hazards ratio, aHR) were quantified by multivariate Cox regression including adjustment for recipient, donor, and transplant clinical factors captured in the OPTN registry. Outcome models were stratified by quintile of propensity for pretransplant narcotic use as previously described [14]. Separate analyses were also performed among the recipients of living donor (LD) and deceased donor (DD) organs.

\section{Results}

\section{Sample Characteristics and Correlates of \\ Pre-Transplant Narcotic Use}

There were 31,197 kidney transplant recipients from 2006 to 2010 with linked transplant registry and pharmacy claims in the year prior to transplantation [14]. Of these, we identified 16,322 recipients $(5,174$ from $L D$ and 11,148 from DD) with linked Medicare claims data from 2006 to 2008 for the current analysis. Twenty-eight percent ( $\mathrm{n}=4,624$ recipients) in this study sample filled a narcotic prescription in the year prior to transplant. Using a priori defined thresholds [14], patients with pretransplant narcotic fills were categorized into four levels of annual ME use: 0.1-1.7 (Level 1, $\mathrm{n}=1,120$ ); 1.8-5.4 (Level 2, $\mathrm{n}=1,144$ ); 5.5-23.7 (Level 3, $\mathrm{n}=1,206$ ); and $\geq 23.8 \mathrm{mg} / \mathrm{kg}$ (Level $4, \mathrm{n}=1,154$ ).

Baseline clinical characteristics according to pretransplant narcotic use level are shown in table 1. Compared with transplant recipients who did not use narcotic medications prior to transplantation, those with Level 4 narcotic use were more often in the age range 45-59, women, white race, not working, publicly insured, and less likely to be college-educated. Recipients with Level 4 pre-transplant narcotic use were also more likely to have longer duration of pre-transplant ESRD and higher rates of sensitization. Distributions of clinical traits according to narcotic use showed similar patterns among LD and DD recipients when considered separately by organ type.

\section{Incidence of Post-Transplant Complications According} to Pre-Transplant Narcotic Use Level

The 3-year incidences of each clinical complication after transplant according to level of pre-transplant narcotic use 
Table 1. Distributions of clinical traits in the study sample of transplant recipients according to pre-transplant narcotic use level

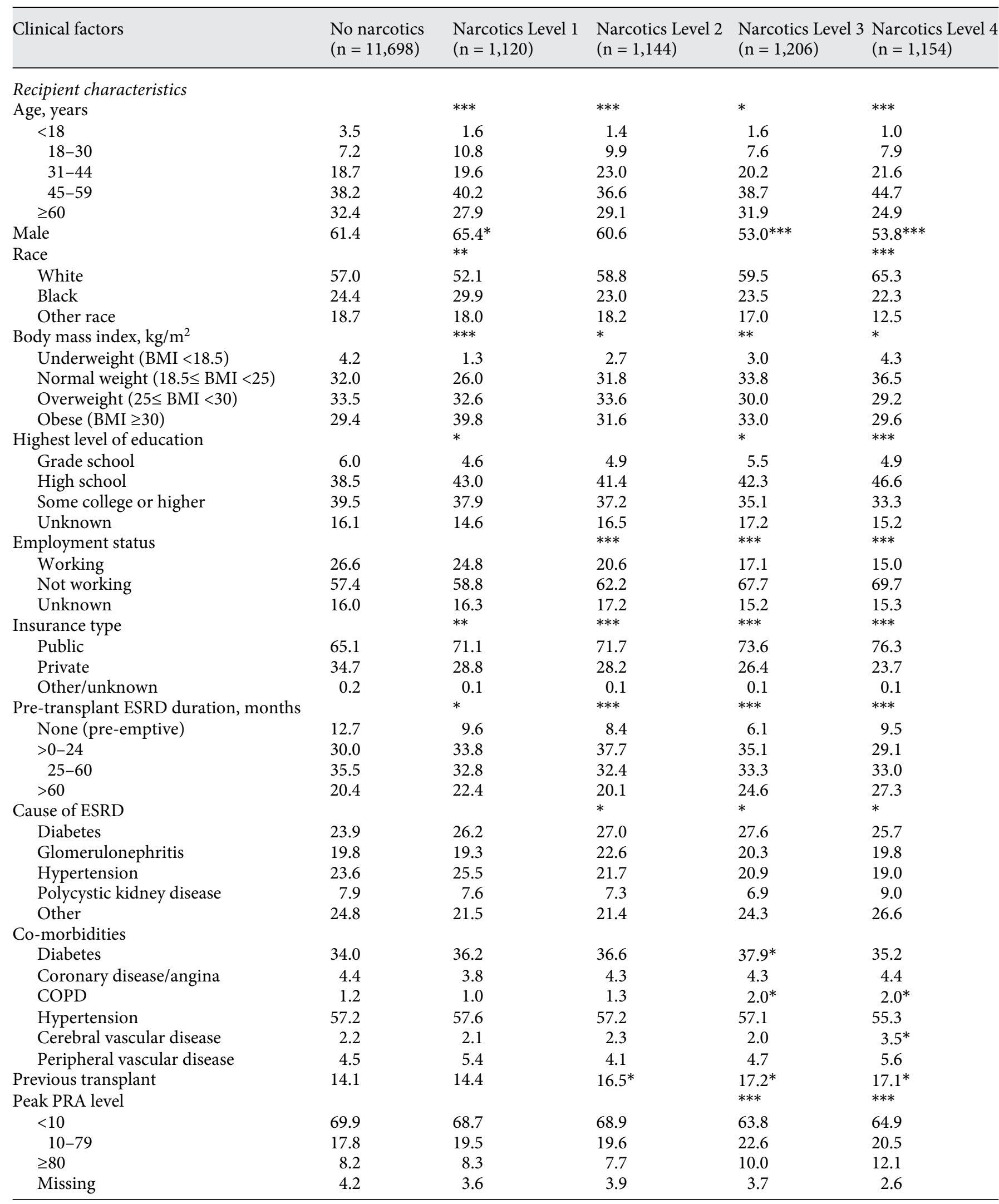


Table 1. (continued)

\begin{tabular}{|c|c|c|c|c|c|}
\hline Clinical factors & $\begin{array}{l}\text { No narcotics } \\
(\mathrm{n}=11,698)\end{array}$ & $\begin{array}{l}\text { Narcotics Level } 1 \\
(\mathrm{n}=1,120)\end{array}$ & $\begin{array}{l}\text { Narcotics Level } 2 \\
(\mathrm{n}=1,144)\end{array}$ & $\begin{array}{l}\text { Narcotics Level } 3 \\
(\mathrm{n}=1,206)\end{array}$ & $\begin{array}{l}\text { Narcotics Level } 4 \\
(\mathrm{n}=1,154)\end{array}$ \\
\hline \multicolumn{6}{|l|}{ Transplant characteristics } \\
\hline Zero A, B, and DR & 10.5 & 10.2 & 12.3 & 11.7 & 12.1 \\
\hline Zero DR & 45.0 & 44.6 & 43.6 & 45.1 & 47.2 \\
\hline Other & 44.5 & 45.2 & 44.1 & 43.2 & 40.7 \\
\hline White & 71.7 & 68.8 & 72.3 & 73.4 & 75.3 \\
\hline Black & 12.2 & 13.5 & 12.9 & 11.8 & 12.5 \\
\hline Other race & 16.1 & 17.8 & 14.8 & 14.8 & 12.2 \\
\hline Female donor & 45.6 & 46.1 & 47.1 & 48.5 & 44.9 \\
\hline CMV sero-pairing & & & & & $*$ \\
\hline Recipient-, Donor- & 15.5 & 14.7 & 16.0 & 13.0 & 13.5 \\
\hline Living & 31.9 & 31.3 & 34.2 & 30.4 & 28.9 \\
\hline Standard criteria deceased & 55.7 & 57.0 & 54.6 & 57.6 & 58.7 \\
\hline Expanded criteria deceased & 12.4 & 11.8 & 11.3 & 11.9 & 12.4 \\
\hline
\end{tabular}

Values are percentages. CMV = Cytomegalovirus; $\mathrm{COPD}=$ chronic obstructive pulmonary disease; ESRD = end-stage renal disease; HLA = human leukocyte antigen; PRA = panel reactive antibody.

$p$ values for differences of distributions of clinical traits among patients in a given narcotic use level compared to those with no pretransplant narcotic use: ${ }^{*} \mathrm{p}<0.05-0.002$; $^{* *} \mathrm{p}=0.001-0.0001 ;{ }^{* *} \mathrm{p}<0.0001$.

are presented in figure 1 . Recipients with Level 4 pre-transplant narcotic use had higher 3-year incidence of posttransplant complications compared to recipients without narcotic use, including ventricular arrhythmias (1.1 vs. $0.2 \%$; $<0.001$ ), cardiac arrest ( 4.7 vs. $2.7 \%$; $p<0.05$ ), hypotension (14 vs. $8 \%$; $\mathrm{p}<0.0001$ ), hypercapnia (1.6 vs. $0.9 \%$; $\mathrm{p}<0.05$ ), mental status changes ( 5.3 vs. $2.7 \%$; $\mathrm{p}<$ 0.001 ), drug abuse/dependence (7.0 vs. $1.7 \%$; $p<0.0001$ ), alcohol abuse ( 1.8 vs. $0.6 \% ; \mathrm{p}=0.0001)$, accidents ( 0.9 vs. $0.3 \%$; $<<0.05)$, and noncompliance ( 3.5 vs. $2.3 \%$; $<<0.05$ ). Aspiration pneumonia was more common among those with Level 3 pre-transplant narcotic use compared with no use.

Patterns were similar when considered separately among recipients of LD and DD transplants (table 2). Compared to LD recipients without pre-transplant narcotic exposure, LD recipients with Level 4 pre-transplant narcotic use more frequently experienced cardiac arrest (5.7 vs. $2.4 \%$; $\mathrm{p}<0.05$ ), hypotension ( 11.7 vs. $6.5 \%$; $\mathrm{p}<$ 0.0001 ), mental status changes ( 3.6 vs. $1.4 \%$; $<<0.05$ ), and drug abuse/dependence ( 7.4 vs. $1.6 \%$; $p<0.0001$ ) by 3 years post-transplant. The frequency of aspiration pneumonia was higher among those with Level 3 pre- transplant narcotic use compared with no use ( 8.5 vs. $0.7 \% ; \mathrm{p}<0.0001)$. Overall, a composite of any of the study complications increased in a graded manner with higher pre-transplant narcotic use levels among LD recipients, reaching statistical significance with Level 3 (20.7\%; p < $0.05)$ and Level $4(25.3 \%$; $p<0.0001)$ pre-transplant narcotic use compared to non-use (12.2\%).

DD recipients with Level 4 pre-transplant narcotic exposure more commonly experienced ventricular arrhythmias ( 1.4 vs. $0.2 \% ; \mathrm{p}<0.0001)$ and noncompliance ( 4.0 vs. $2.5 \% ; \mathrm{p}<0.05)$ by 3 years post-transplant compared to those who did not use prescription narcotic agents before transplant. Further, significant graded increases in the post-transplant complications beginning with Level 3 pre-transplant narcotics exposure were observed for hypotension (non-use: 9.4\%, Level 3: 12.6\%, Level 4: 15.0\%), mental status changes (non-use: 3.3\%, Level 3: 4.7\%, Level 4: $6.0 \%$ ), drug abuse/dependence (non-use: $1.8 \%$, Level 3: 3.3\%, Level 4: 6.8\%), alcohol abuse (non-use: 0.8\%, Level 3: $1.4 \%$, Level $4: 2.1 \%$ ), and accidents (non-use: $0.3 \%$, Level 3: $1.1 \%$, Level 4: $1.3 \%)$. Compared to non-users, the 3 -year incidence of cardiac arrest was increased to a similar level among recipients with Level 3 (4.1 vs. $2.8 \%$; $\mathrm{p}<$ 
Fig. 1. Three-year incidence of post-transplant complications according to pretransplant narcotic use level in the full study sample. ${ }^{*} \mathrm{p}<0.05-0.0001 ;{ }^{\ddagger} \mathrm{p}<$ 0.0001 .

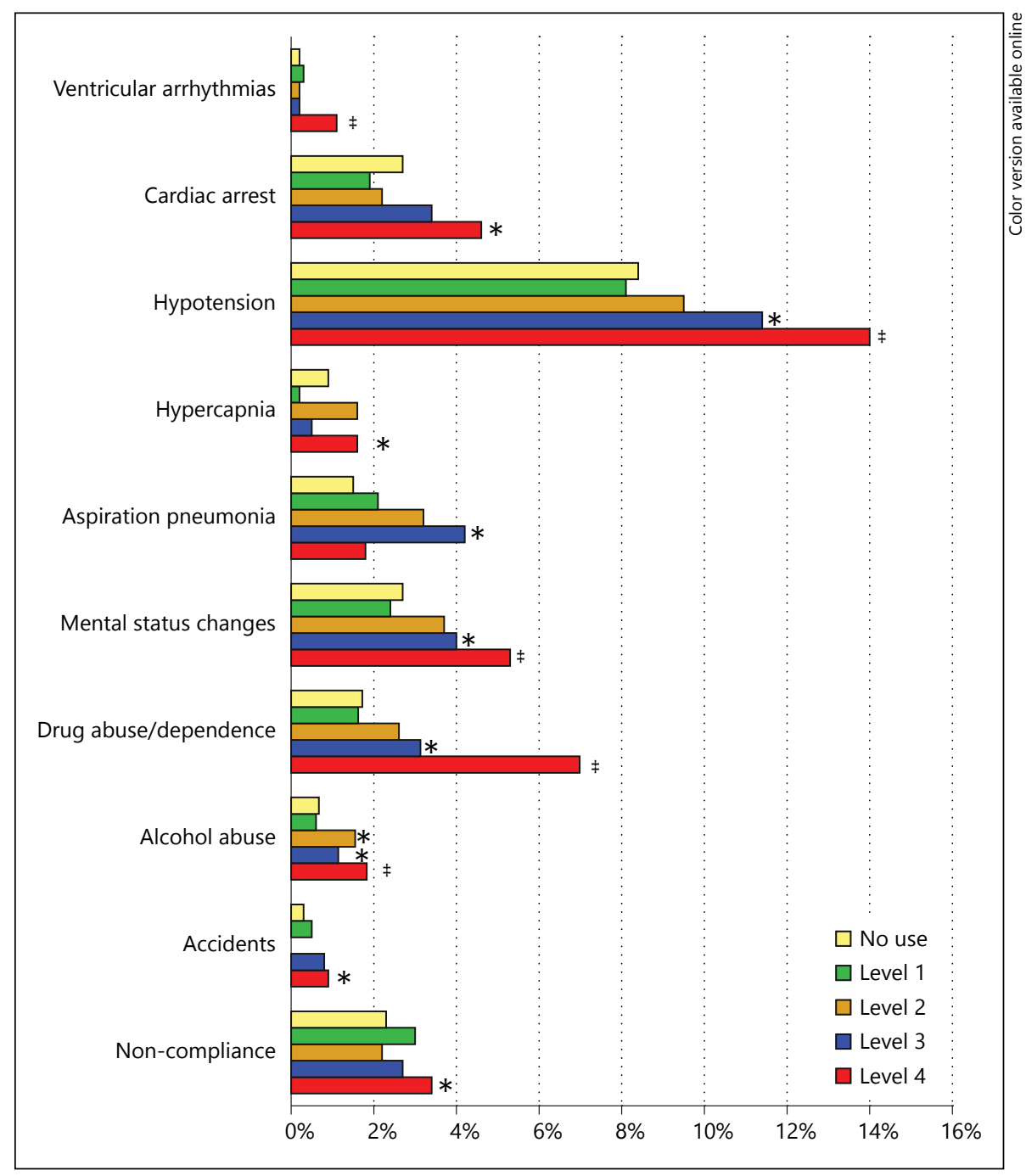

$0.05)$ and Level 4 (4.1 vs. $2.8 \%$; $<$ < 0.05) pre-transplant ME. Patterns for hypercapnia and aspiration pneumonia were inconsistent and not significant. Overall, in DD recipients, the 3-year incidence of a composite of any study complications was $22.6 \%(\mathrm{p}<0.05)$ and $29.6 \%(\mathrm{p}<0.0001)$ among those with Level 3 and Level 4 pre-transplant ME use, respectively, compared with $17.7 \%$ among non-users.

\section{Adjusted Associations of Pre-Transplant Narcotic Use with Post-Transplant Complications}

After multivariate adjustment for recipient, donor, and transplant factors, as well as propensity adjustment for the likelihood of pre-transplant narcotic use, transplant recipients with Level 4 pre-transplant ME use had approximately 2 to 4 times the risk of post-transplant ventricular arrhythmias (aHR 3.92; 95\% CI 1.74-8.84), mental status changes (aHR 2.03; 95\% CI 1.46-2.83), drug abuse/dependence (aHR 3.17; 95\% CI 2.35-4.28), alcohol abuse (aHR $2.41 ; 95 \%$ CI 1.38-4.20), and accidents (aHR 3.09; 95\% CI 1.28-7.45) compared with non-users (fig. 2). Recipients with Level 4 pre-transplant narcotic use also had $43 \%$ higher likelihood of post-transplant cardiac arrest (aHR $1.43 ; 95 \%$ CI $1.00-2.05)$ and a $35 \%$ relative increase in hypotension (aHR 1.35; 95\% CI 1.10-1.65). A trend for increased noncompliance diagnoses for those with Level 4 pre-transplant narcotic use was nearly significant (aHR $1.51 ; 95 \%$ CI $0.99-2.29 ; \mathrm{p}=0.06$ ). Overall, the adjusted 3 -year risk of any study complication was increased by $61 \%$ among transplant recipients with Level $4 \mathrm{ME}$ use compared to non-users (aHR 1.61; 95\% CI 1.41-1.85). Complete regression results for associations of pretransplant narcotic use and baseline recipient, donor and 
Table 2. Three-year incidence of post-transplant complications according to pre-transplant narcotic use level, by transplant donor type

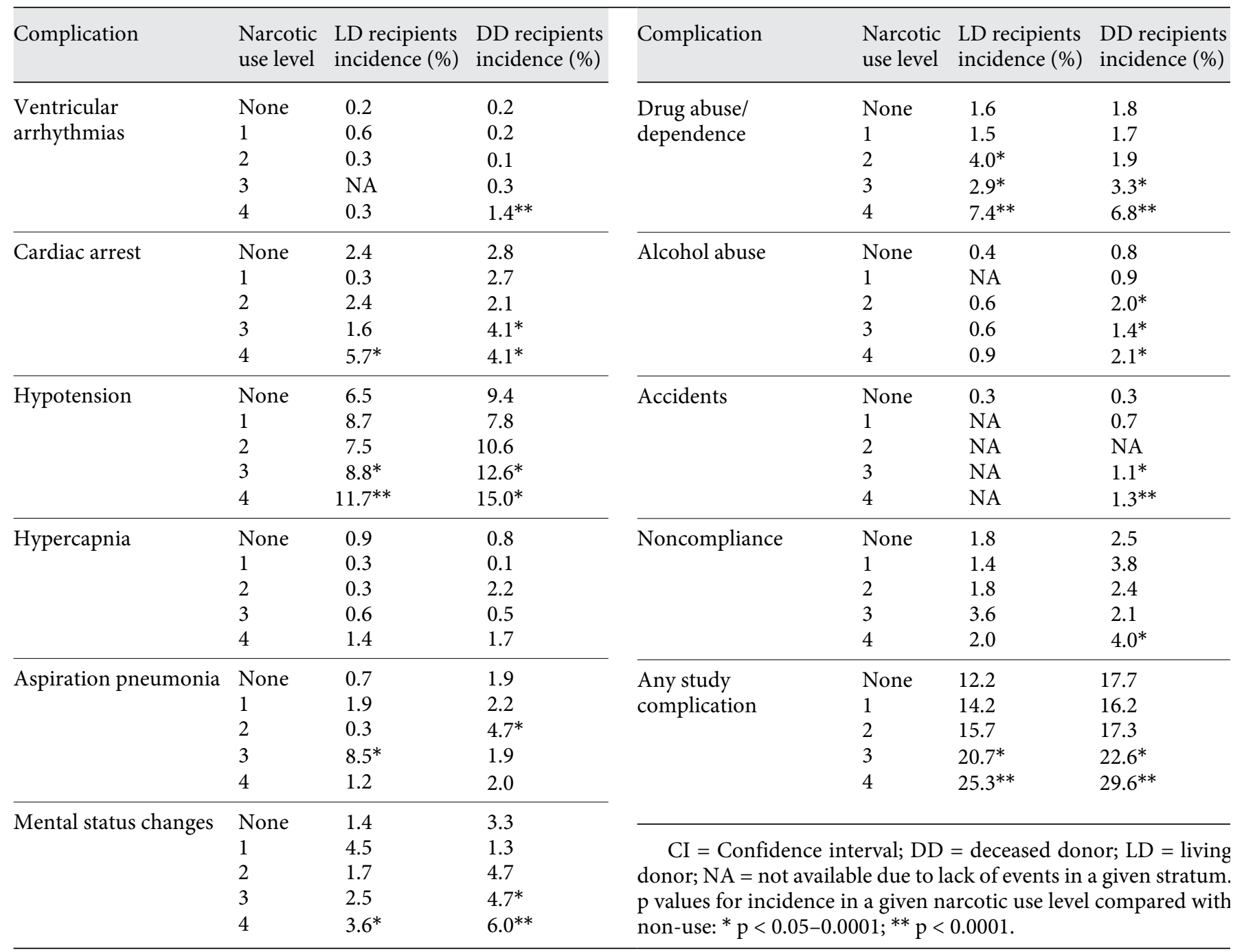

transplant factors with the composite outcome are provided in online supplementary appendix 2 .

LD transplant recipients with Level 3 pre-transplant ME use had higher adjusted risk of aspiration pneumonia (aHR 5.96; 95\% CI 2.61-13.6) compared to non-users, although pneumonia patterns were not graded across other levels (table 3). LD recipients with Level 4 pretransplant ME use had 69\% higher adjusted risk of posttransplant hypotension (aHR 1.69; 95\% CI 1.16-2.46), 2.7 times the risk of mental status changes (aHR 2.68; 95\% CI 1.24-5.77), and 4 times the risk of drug abuse/dependence (aHR 4.06; 95\% CI 2.38-6.93). The adjusted 3-year risk of any study complication was increased by $83 \%$ among LD transplant recipients with Level $4 \mathrm{ME}$ use compared to non-users (aHR 1.83; 95\% CI 1.40-2.38).
Pre-Transplant Narcotic Use and Complications
Among DD transplant recipients, significant increases in the adjusted risk of post-transplant complications beginning with Level 3 pre-transplant ME exposure were observed for mental status changes (Level 3: aHR 1.53; 95\% CI 1.04-2.25, Level 4: 1.92; 95\% CI 1.33-2.78), drug abuse/dependence (Level 3: aHR 1.61; 95\% CI 1.02-2.54, Level 4: aHR 2.79; 95\% CI 1.94-4.02), and alcohol abuse (Level 3: aHR 2.04; 95\% CI 1.05-3.97, Level 4: aHR 2.39; 95\% CI 1.31-4.38). DD recipients with Level 4 pre-transplant ME use also had significantly increased adjusted risk of ventricular arrhythmias (aHR 5.58; 95\% CI 2.1914.21), accidents (aHR 4.73; 95\% CI 1.85-12.1), and noncompliance diagnoses (aHR 1.76; 95\% CI 1.11-2.79). Although there was a trend toward increased risk of hypotension among DD transplant recipients with higher 
Fig. 2. Adjusted associations of pre-transplant narcotic use with post-transplant complications in the full study sample (adjusted for recipient, donor, and transplant factors in table 1).

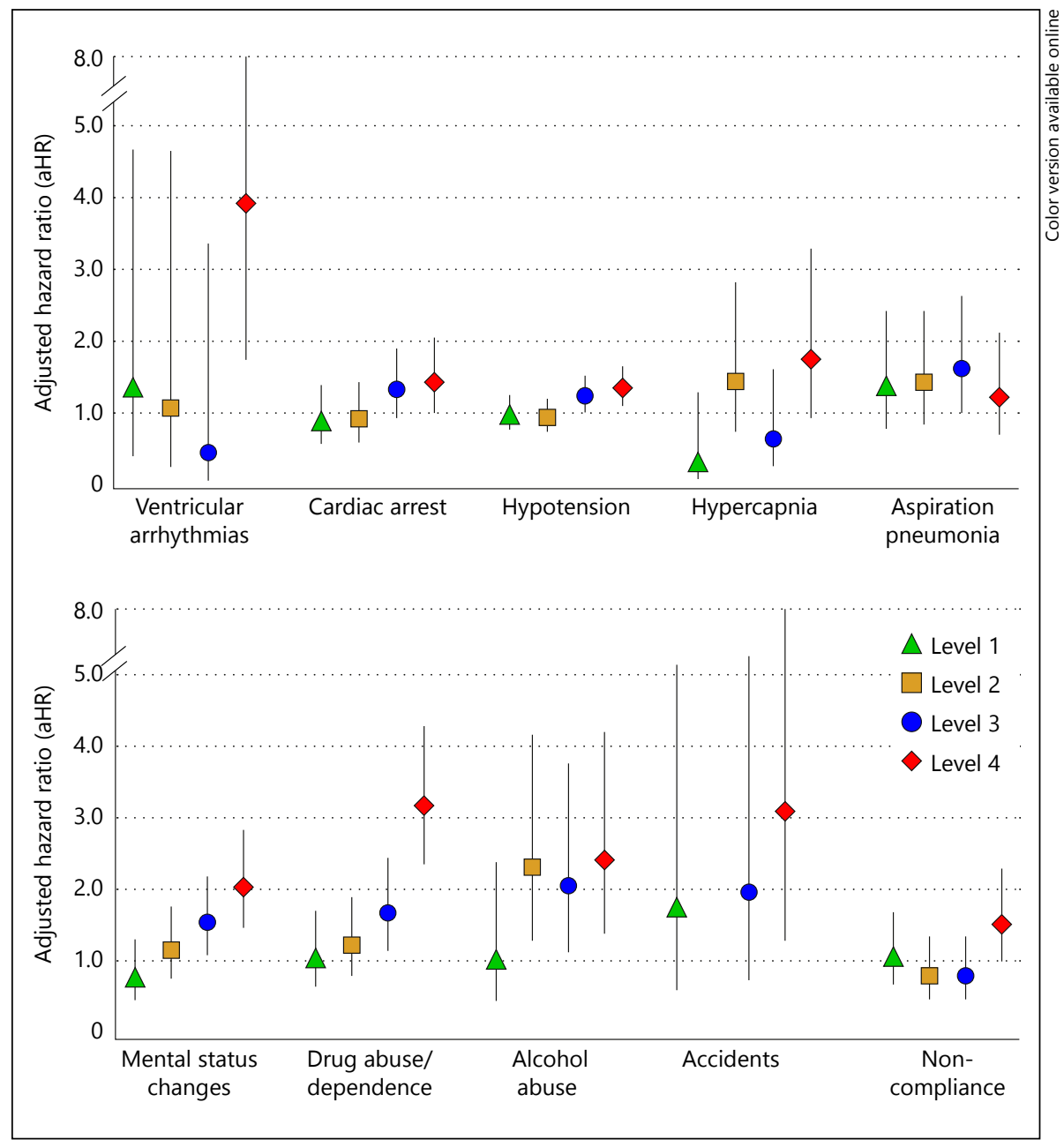

pre-transplant narcotic exposure, this pattern did not reach statistical significance. The adjusted risk of any study complication was increased by $26 \%$ (aHR 1.26; 95\% CI 1.07-1.49) and 56\% (aHR 1.56; 95\% CI 1.33-1.82) in DD transplant recipients with Level 3 and Level 4 pretransplant ME use compared to non-users, respectively.

\section{Discussion}

Public health advocates have highlighted an epidemic of both use and complications of prescription opioid analgesics in the United States and Canada, two of the highest prescription opioid consumers in the world $[1,2,15]$. In the United States, narcotic prescriptions have increased $300 \%$ from 1999 to 2010 [16]. In this context, the annual rate of documented deaths from accidental narcotic overdoses increased from 4,000 to nearly 17,000
[17]. One case-cohort study of the Veterans Health Administration found that the frequency of fatal opioid overdoses was directly related to the maximum prescribed daily dose of opioid medication [4]. Recently, the Centers for Disease Control and Prevention (CDC) issued a press release emphasizing concern for the public health impacts of prescription opioid painkillers, noting that deaths involving such painkillers were much more common than heroin-related deaths [18]. Underlying mechanisms of opioid analgesic-related fatal and non-fatal complications include cardiac arrhythmias, respiratory depression, mental status changes, and aberrant behaviors resulting in accidents and overall poor self-care. For example, in a retrospective cohort study of older patients receiving opioid therapy in the United States, adverse events occurred in $40 \%$ of cases, including mental status changes (16\%), lethargy (9\%), and abuse/misuse behaviors (3\%) [19]. Reflecting concerns for common misuse 
Table 3. Adjusted associations of pre-transplant narcotic use with post-transplant complications by transplant donor type (adjusted for recipient, donor, and transplant factors in table 1)

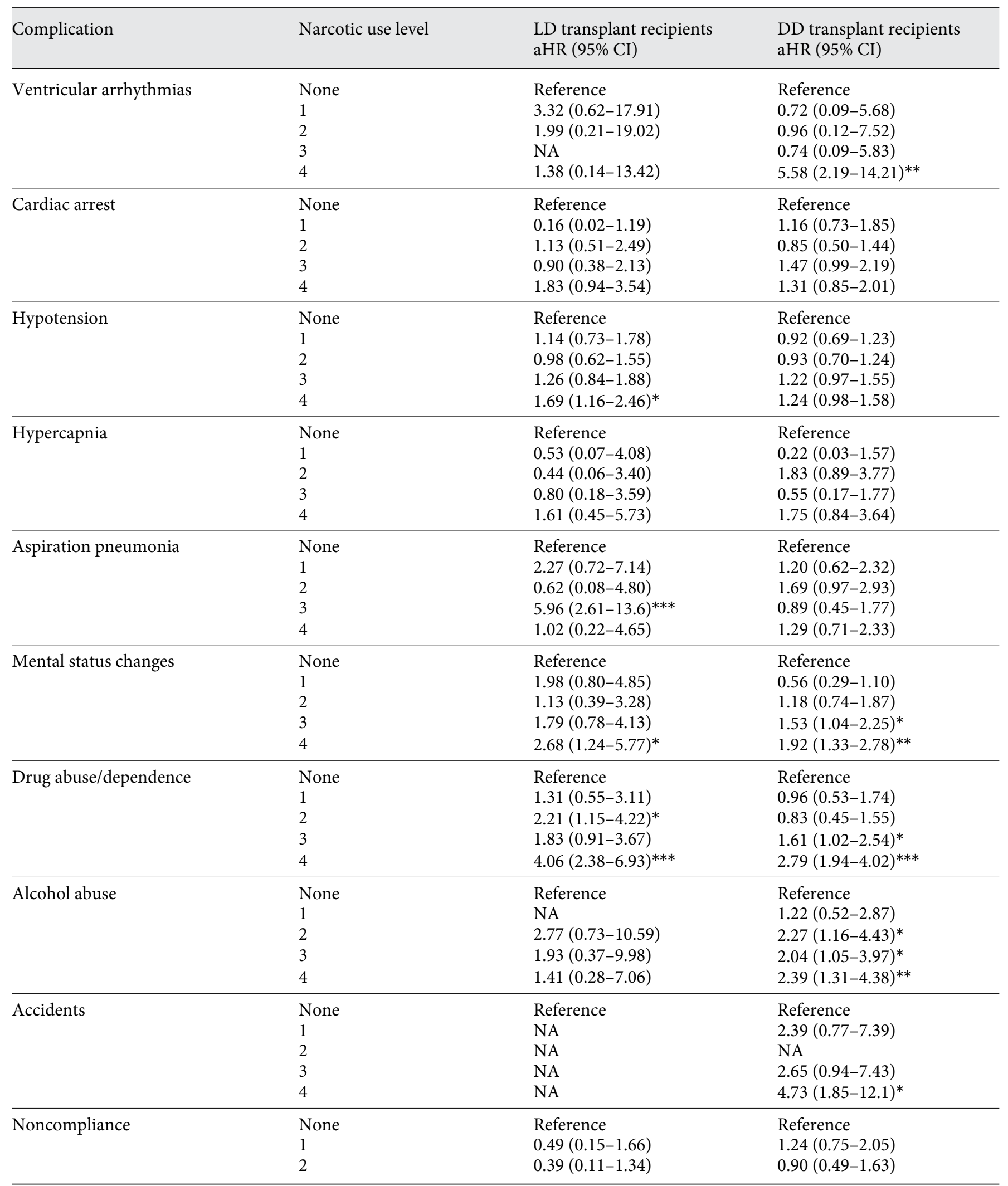


Table 3. (continued)

\begin{tabular}{llll}
\hline Complication & Narcotic use level & $\begin{array}{l}\text { LD transplant recipients } \\
\text { aHR (95\% CI) }\end{array}$ & $\begin{array}{l}\text { DD transplant recipients } \\
\text { aHR (95\% CI) }\end{array}$ \\
\hline & 3 & $0.50(0.15-1.67)$ & $0.87(0.48-1.58)$ \\
& 4 & $0.85(0.29-2.48)$ & Reference \\
\hline Any study complication & None & Reference & $0.96(0.78-1.17)$ \\
& 2 & $1.00(0.71-1.41)$ & $1.03(0.85-1.24)$ \\
$1.26(1.07-1.49)^{*}$ & $1.18(0.87-1.60)$ & $1.56(1.33-1.82)^{* *}$ \\
\hline
\end{tabular}

aHR = Adjusted hazard ratio; $\mathrm{DD}=$ deceased donor; $\mathrm{LD}=$ living donor; $\mathrm{NA}=$ not available due to lack of events in a given stratum. p values: ${ }^{*} \mathrm{p}<0.05-0.002 ;{ }^{* *} \mathrm{p}=0.001-0.0001$; $^{* *} \mathrm{p}<0.0001$.

and complications of opioid pain medications, the Food and Drug Administration (FDA) Drug Safety and Risk Management Advisory Committee recently recommended re-classifying hydrocodone-containing products from Schedule III to the more restrictive Schedule II level of controlled substances [20].

To date, there is little information available on the clinical outcomes of narcotic use among kidney transplant patients. A single center study recently found that pretransplant opioid use identified from chart review was associated with post-transplant death [13], while we recently found associations of pre-transplant narcotic use defined by pharmacy fill records with increased risks of post-transplant death and graft loss in a larger national sample [14]. In the current study, we expanded our linkage of national transplant registry data and outpatient pharmacy fill records to incorporate Medicare billing claims as measures of clinical conditions diagnosed after transplantation. To our knowledge, this is the first study to identify a connection between narcotic use and relevant clinical complications in the kidney transplant population, specifically, cardiovascular (ventricular arrhythmias, cardiac arrest, hypotension), respiratory (hypercapnia), neurological (mental status changes), substance abuse, accidents, and noncompliance events. In adjusted analyses, transplant recipients with the highest level pretransplant narcotic use had approximately 2 to 4 times the risks of post-transplant ventricular arrhythmias, mental status changes, drug abuse/dependence, alcohol abuse, and accidents compared with non-users, and approximately 35 to $45 \%$ higher likelihood post-transplant cardiac arrest and hypotension. Overall, compared to non-users, LD recipients with the highest level of pretransplant narcotic exposure use had $83 \%$ increased rela- tive risk of a composite of any study complication, while DD recipients had a $56 \%$ increased risk of any clinical complication over 3 years.

For transplant recipients, compliance with medical care is of particular concern and includes the need to adhere to immunosuppression therapy and other transplant-related medications, perform regular lab testing and attend follow-up appointments [21,22]. Noncompliance among transplant recipients is often difficult to diagnose, but when recognized, it has been shown to correlate strongly with preventable allograft loss and poor patient outcomes [23-26]. While diagnostic codes for noncompliance were found in less than $5 \%$ of our study sample, we suspect that clinically coded noncompliance is an underestimate and that more subtle cases are not captured in diagnostic claims. Our results suggest that high-level pre-transplant narcotic use is a marker for patients with increased risk for noncompliance after transplantation. It is likely that drug and alcohol abuse are also underreported to providers and underestimated in billing claims, but it is notable that we detected significant associations between high-level pre-transplant narcotic use and diagnoses of post-transplant substance abuse. This correlation of prescription narcotic use and other substance abuse problems is consistent with a recent study reporting that the most important risk factor for opioid dependence, abuse and overdose in the general population was not a feature of the individual patient, but rather use of a prescription narcotic medication [17]. Given the potential biases in self-reported narcotic use, prescription fill records may be a useful tool for determining narcotic use patterns before and after transplantation. However, further study is needed to identify strategies that may be effective in improving compliance and reduc- 
ing complications in transplant recipients requiring high levels of pre-transplant narcotic medications.

Our study has strengths, including the use of a novel linkage of large databases to investigate the outcomes of pre-transplant medication exposure. Electronic pharmacy claims and fill records have been shown to be highly accurate records of physician prescriptions that circumvent some of the limitations of self-reported medication use [27-29]. However, there were also important limitations to our study. Due to the observational nature of our study design, we were able to describe associations but were not able to prove causality. Unmeasured factors, including patient behaviors, may affect the outcomes described. Although electronic pharmacy claims and fill records have been found to be highly accurate records of physician prescribing, we were unable to account for illicit drug use or over-the-counter medication use. The degree of opioid exposure may be underestimated if details about patients engaged in 'pharmacy shopping' behaviors who filled narcotic prescriptions at other pharmacies were not captured in our database; however, these patients would likely be categorized as high-level users if they submitted the majority of their narcotic prescriptions at the same pharmacy where they filled their immunosuppression prescriptions. Additionally, physical examination measurements (such as blood pressure readings), laboratory values (such as arterial blood gas results), and diagnostic test results (such as electrocardiograms and chest X-rays) were not available to adjudicate the clinical outcomes in our study. Since we relied on physician diagnoses, diagnostic codes, and outpatient prescriptions to define our outcomes and primary exposure, there is a possibility of misclassification. Lastly, it may be that recipients with a known history of chronic opioid use are more likely to be diagnosed with opioidrelated adverse events than non-users (i.e., ascertainment bias).

Despite these limitations, our study illustrated that the integration of national transplant registry, pharmacy fill records, and medical billing claims databases can enable examination of new risk factors for clinical complications after kidney transplantation. Risk stratification is particularly important in the era of public reporting and regulatory scrutiny of transplant recipient patient and graft survival. Notably, the risk-adjusted equations developed by the Scientific Registry of Transplant Recipients (SRTR) for the prediction of expected 1-year and 3-year posttransplant patient and graft survival in the United States do not adjust for narcotic use, pain, or compliance behaviors [30]. Our a priori study design is based on pre-trans- plant narcotic use, a relevant exposure given that regulatory risk assessment considers pre-transplant factors. However, future studies should also examine the outcome implications of narcotic use after kidney transplantations.

In conclusion, we found that high-level opioid analgesic use before kidney transplantation is associated with adverse clinical events after transplantation, including cardiovascular, respiratory, neurological, substance abuse, and compliance complications. Further research is needed to identify kidney transplant recipients who are most likely to experience narcotic-related complications after transplantation and management strategies to mitigate risks of these complications. Currently, we recommend that kidney transplant candidates with moderate-tosevere pain undergo a careful evaluation of pain management strategies utilizing a multidisciplinary team approach, possibly including pain management specialists.

\section{Acknowledgments}

The data reported here has been supplied by the United Network for Organ Sharing (UNOS) as the contractor for the Organ Procurement and Transplantation Network (OPTN). This work was supported by grants from the National Institutes of Health (NIH)/National Institute of Diabetes and Digestive and Kidney Diseases (NIDDK) R01-DK102981 and RC1-DK86450. NNL was supported by the Clinical Investigator Program at Western University and by a Kidney Research Scientist Core Education and National Training Program (KRESCENT) postdoctoral fellowship award. The interpretation and reporting of this data are the responsibility of the author(s) and in no way should be seen as an official policy of or interpretation by the OPTN, the United States Government, or the funding sources.

\section{Disclosure Statement}

The authors have no conflicts of interest related to this work. This work was supported by grants from the National Institutes of Health (NIH)/National Institute of Diabetes and Digestive and Kidney Diseases (NIDDK) R01DK102981 and RC1-DK86450.

References

Manchikanti L, Helm S, Fellows B, Janata JW, Pampati V, Grider JS, et al: Opioid epidemic in the United States. Pain Physician 2012;15: ES9-ES38.

$\checkmark 2$ Betses M, Brennan T: Abusive prescribing of controlled substances - a pharmacy view. $\mathrm{N}$ Engl J Med 2013;369:989-991.

-3 Kalso E, Edwards JE, Moore RA, McQuay HJ Opioids in chronic non-cancer pain: systematic review of efficacy and safety. Pain 2004; 112:372-380. 
4 Bohnert AS, Valenstein M, Bair MJ, Ganoczy D, McCarthy JF, Ilgen MA, et al: Association between opioid prescribing patterns and opioid overdose-related deaths. JAMA 2011;305: 1315-1321.

5 Rice JB, White AG, Birnbaum HG, Schiller M, Brown DA, Roland CL: A model to identify patients at risk for prescription opioid abuse, dependence, and misuse. Pain Med 2012;13: 1162-1173.

-6 Salisbury EM, Game DS, Al-Shakarchi I, Chan M, Fishman L, Tookman L, et al: Changing practice to improve pain control for renal patients. Postgrad Med J 2009;85:30-33.

$\checkmark 7$ Weisbord SD, Fried LF, Arnold RM, Fine MJ, Levenson DJ, Peterson RA, et al: Prevalence, severity, and importance of physical and emotional symptoms in chronic hemodialysis patients. J Am Soc Nephrol 2005;16:2487-2494.

$\checkmark 8$ Davison SN: Pain in hemodialysis patients: prevalence, cause, severity, and management. Am J Kidney Dis 2003;42:1239-1247.

-9 Barakzoy AS, Moss AH: Efficacy of the world health organization analgesic ladder to treat pain in end-stage renal disease. J Am Soc Nephrol 2006;17:3198-3203.

10 Dean M: Opioids in renal failure and dialysis patients. J Pain Symptom Manage 2004;28: 497-504.

11 Kurella M, Bennett WM, Chertow GM: Analgesia in patients with ESRD: a review of available evidence. Am J Kidney Dis 2003;42:217228.

12 Masajtis-Zagajewska A, Pietrasik P, Krawczyk J, Krakowska M, Jarzębski T, Pietrasiewicz B, et al: Similar prevalence but different characteristics of pain in kidney transplant recipients and chronic hemodialysis patients. Clin Transplant 2011;25:E144-E151.

13 Barrantes F, Luan FL, Kommareddi M, Alazem K, Yaqub T, Roth RS, et al: A history of chronic opioid usage prior to kidney trans- plantation may be associated with increased mortality risk. Kidney Int 2013;84:390-396.

14 Lentine KL, Yuan H, Tuttle-Newhall JE, Xiao $\mathrm{H}$, Chawa V, Axelrod D, et al: Quantifying prognostic impact of prescription opioid use before kidney transplantation through linked registry and pharmaceutical claims data. Transplantation 2015;99:187-196.

15 Gomes T, Mamdani MM, Paterson JM, Dhalla IA, Juurlink DN: Trends in high-dose opioid prescribing in Canada. Can Fam Physician 2014;60:826-832.

16 Kunins HV, Farley TA, Dowell D: Guidelines for opioid prescription: why emergency physicians need support. Ann Intern Med 2013; 158:841-842.

17 Dowell D, Kunins HV, Farley TA: Opioid analgesics - risky drugs, not risky patients. JAMA 2013;309:2219-2220.

18 Centers for Disease Control and Prevention: Heroin overdose deaths increased in many states through 2012. http://www.cdc.gov/ media/releases/2014/p1002-heroin-overdose.html (cited October 4, 2014).

19 Reid MC, Henderson CR Jr, Papaleontiou M, Amanfo L, Olkhovskaya Y, Moore AA, et al: Characteristics of older adults receiving opioids in primary care: treatment duration and outcomes. Pain Med 2010;11:1063-1071.

20 Shots - Health News NPR: FDA seeks to tighten controls on hydrocodone painkillers. http://www.npr.org/blogs/health/2013/10/ 24/240569516/fda-seeks-to-tighten-controlson-hydrocodone-painkillers (cited October 4, 2014).

21 Williams AF, Manias E, Gaskin CJ, Crawford $\mathrm{K}$ : Medicine non-adherence in kidney transplantation. J Ren Care 2014;40:107-116.

22 Denhaerynck K, Steiger J, Bock A, SchäferKeller P, Köfer S, Thannberger N, et al: Prevalence and risk factors of non-adherence with immunosuppressive medication in kidney transplant patients. Am J Transplant 2007;7: 108-116.

23 Lerut E, Kuypers DR, Verbeken E, Cleutjens J, Vlaminck H, Vanrenterghem Y, et al: Acute rejection in non-compliant renal allograft recipients: a distinct morphology. Clin Transplant 2007;21:344-351.

-24 Vlaminck H, Maes B, Evers G, Verbeke G, Lerut E, Van Damme B, et al: Prospective study on late consequences of subclinical non-compliance with immunosuppressive therapy in renal transplant patients. Am J Transplant 2004;4:1509-1513.

25 De Geest S, Borgermans L, Gemoets H, Abraham I, Vlaminck H, Evers G, et al: Incidence, determinants, and consequences of subclinical noncompliance with immunosuppressive therapy in renal transplant recipients. Transplantation 1995;59:340-347.

26 Pinsky BW, Takemoto SK, Lentine KL, Burroughs TE, Schnitzler MA, Salvalaggio PR: Transplant outcomes and economic costs associated with patient noncompliance to immunosuppression. Am J Transplant 2009;9: 2597-2606.

27 Levy AR, O’Brien BJ, Sellors C, Grootendorst P, Willison D: Coding accuracy of administrative drug claims in the Ontario drug benefit database. Can J Clin Pharmacol 2003;10: 67-71.

28 Lau HS, de Boer A, Beuning KS, Porsius A: Validation of pharmacy records in drug exposure assessment. J Clin Epidemiol 1997;50: 619-625.

29 Boethius G: Recording of drug prescriptions in the county of Jämtland, Sweden. II. Drug exposure of pregnant women in relation to course and outcome of pregnancy. Eur J Clin Pharmacol 1977;12:37-43.

30 SRTR: Risk-adjustment models. http://www. srtr.org/csr/current/modtabs.aspx (cited October 4, 2014). 